\title{
Recherches sur l'anatomie et la biologie de Microcotyle salpae Parona et Perugia, 1890 parasite de Box salpa L. (Téléostéen)
}

\author{
par M.-H. KTARI
}

[Faculté de Sciences de Tunis et I.N.S.T.O.P., Salammbô (Tunisie)]

\section{Résumé}

La morphologie et l'anatomie de Microcotyle salpae trouvé à Tunis chez Box salpa sont redécrites. La localisation de ces parasites sur les branchies est précisée. Les larves non ciliées ont été obtenues et on donne leur anatomie. L'influence de l'hôte sur les modalités de l'éclosion et de l'infestation est dégagée expérimentalement.

\section{Summary}

The morphology and anatomy of Microcotyle salpae from Box salpa found in Tunis, are described anew. The position of these parasites on the gills is precised. Unciliated larvae have been obtained and their anatomy is given. The influence of the host upon the modalities of the hatching and infestation has been brought out experimentally.

Microcotyle salpae a été brièvement décrit par Parona et Perugia dans leur travail de 1890. Cette description est basée sur l'étude d'un seul individu récolté sur les branchies de Box salpa à Gênes. L'espèce a depuis été citée par Mac Callum (1913), Méserve (1938), Sproston (1946), Palombi (1949), Yamaguti (1963); mais tous ces auteurs se sont contentés de reprendre les quelques données fournies par les auteurs italiens. Ainsi, Microcotyle salpae, qui ne paraît pas avoir été revu depuis la description originale, reste une espèce très mal connue. Nous avons récolté sur les branchies de Saupes (Box salpa) pêchées dans le golfe de Tunis, un Monogène qui correspond à Microcotyle salpae. La description de ce parasite étant, nous l'avons dit, des plus suc- 

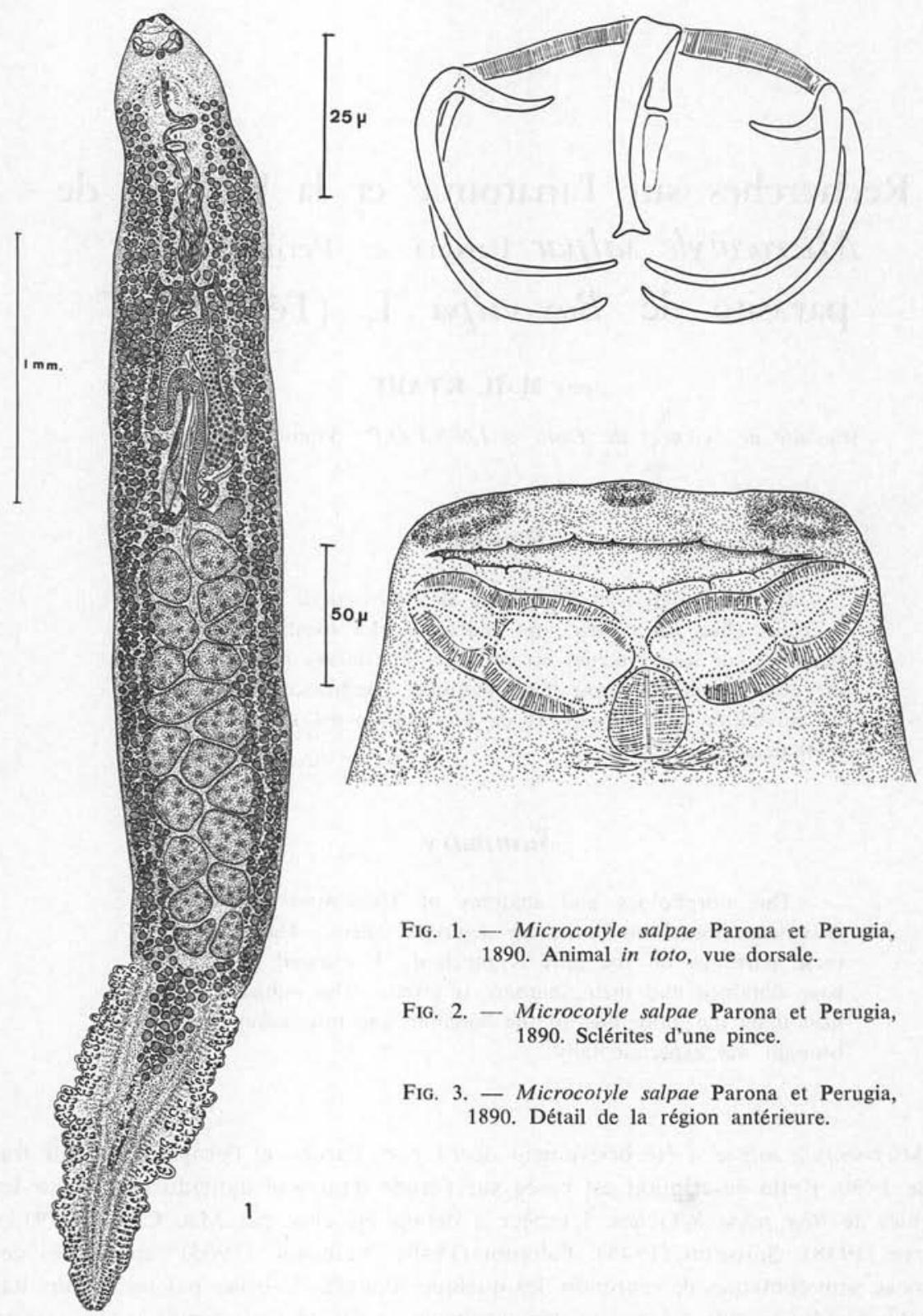

Fig. 1. - Microcotyle salpae Parona et Perugia, 1890. Animal in toto, vue dorsale.

Fig. 2. - Microcotyle salpae Parona et Perugia, 1890. Sclérites d'une pince.

Fig. 3. - Microcotyle salpae Parona et Perugia, 1890. Détail de la région antérieure.

cinctes et son iconographie des plus réduites (un seul schéma de l'atrium génital), nous croyons nécessaire de compléter les données actuelles. Nous ajouterons quelques observations sur la biologie particulière de ce Monogène. 
Microcotyle salpae Parona et Perugia, 1890.

Hôte: Box salpa L. (Sparidae).

HaBITAT : Branchies.

Localité : Tunis.

Matériel ÉTUdié : 75 individus colorés et montés in toto, 5 individus débités en coupes sériées transversales et longitudinales.

\section{Description.}

\section{Morphologie.}

Les individus que nous avons récoltés mesurent de 3 à $5 \mathrm{~mm}$ de longueur et de 0,4 à $0,6 \mathrm{~mm}$ de largeur. Une légère construc.ion située au niveau du tiers postérieur de l'animal sépare le hapteur du corps proprement dit (fig. 1). A la partie antérieure amincie, tronquée à son extrémité, débouchent trois amas glandulaires (un petit amas central et deux latéraux plus importants). L'ouverture buccale est subterminale ventrale. L'atrium génital s'ouvre ventra'ement à $300 \mu$ environ de l'extrémité antérieure. Le vagin dorsal est situé sur la ligne médiane à $200 \mu$ environ de l'atrium. Le hapteur grossièrement triangulaire mesure de 0,85 à $1 \mathrm{~mm}$ de longueur. La largeur varie de $425 \mu$ près du corps à $80 \mu$ près de l'extrémité postérieure. Il présente de chaque côté une rangée de 48 à 50 pinces; il y a donc en tout de 96 à 100 pinces disposées en 48-50 paires symétriques. Ces pinces possèdent des pièces sclérifiées dont la disposition est identique à celle que l'on rencontre chez les autres espèces du genre Microcotyle et notamment à celle de l'espèce type Microcotyle donavini Van Beneden et Hesse, 1863 (Euzet et Marc, 1963) (fig. 2). Les pinces terminales mesurent $35 \mu$ sur $25 \mu$. Les pinces augmentent régulièrement de taille et atteignent $80 \mu$ sur $50 \mu$ dans le milieu de chaque file.

\section{Anatomie.}

\section{TUBE DIGESTIF :}

A la bouche fait suite une large cavité buccale dans laquelle s'ouvre de chaque côté une ventouse musculaire de $90 \mu$ sur $45 \mu$. Chacune de ces ventouses est subdivisée en deux logettes, légèrement inégales, par un septe musculaire transversal situé à 40 ॥ environ du bord latéral. Le rebord de ces ventouses est marqué par une rangée régulière d'une centaine de minuscules papilles coniques (fig. 3). Chaque rangée est interrompue au niveau du pharynx musculeux médian qui mesure $30 \mu$ environ de diamètre. L'œsophage qui lui fait suite, rectiligne, de $90 \mu$ de longueur, se divise au niveau de l'atrium génital en deux branches latérales qui présentent de nombreux caecums latéraux et axiaux. Les deux branches s'unissent à la partie postérieure du corps et forment un seul diverticule médian qui pénètre assez profondément dans le hapteur. 
ApPareil géNital Male :

Dans la moitié postérieure du corps les testicules au nombre de 13 à 20 sont disposés suivant deux lignes longitudinales étroitement imbriquées et paraissent comprimées latéralement par les caecums intestinaux et les glandes vitellogènes. Les testicules les plus antérieurs allongés transversalement mesurent environ $100 \mu$ sur $50 \mu$. Ils sont de plus en plus petits et circulaires vers l'arrière, les derniers ayant $50 \mu$ environ de diamè-

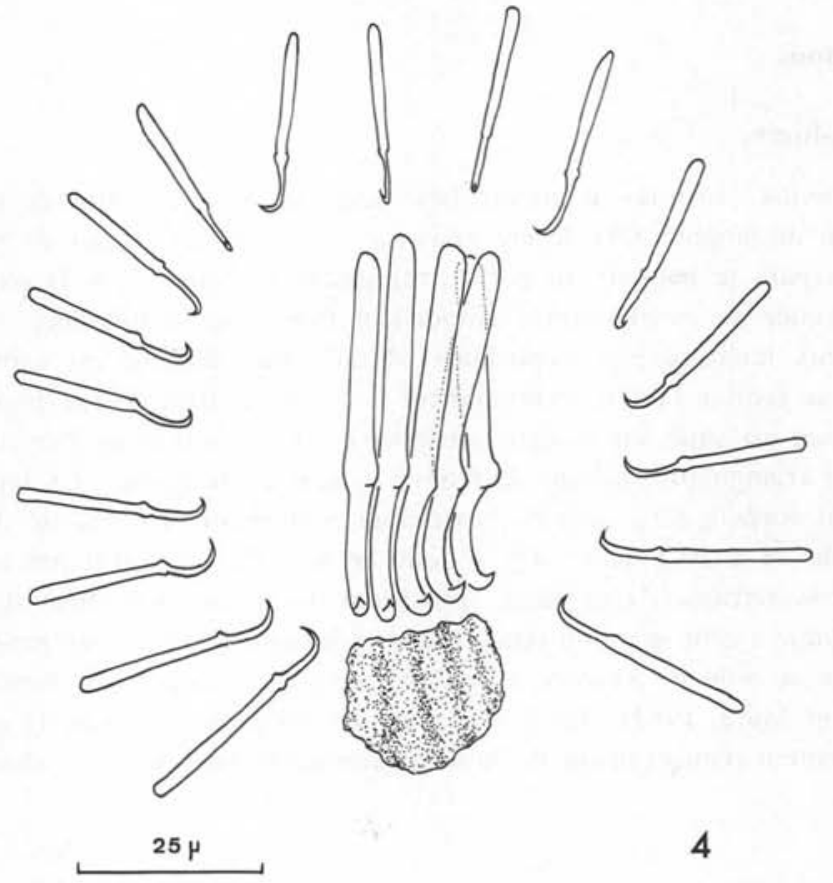

Fig. 4. - Microcotyle salpae Parona et Perugia, 1890. Armement de l'atrium génital.

tre. Le canal déférent médian se dirige vers l'avant. D'abord rectiligne et étroit, il devient sinueux en avant de l'ovaire et dans cette partie, où il est, en général, bourré de spermatozoïdes, il mesure jusqu'à $35 \mu$ de diamètre. Le canal déférent se rétrécit au niveau de l'atrium génital qu'il contourne dorsalement sur la ligne médiane. Il forme a!ors, en avant, un coude et vient déboucher dans la partie antéro-dorsale de l'atrium génital. Cet atrium grossièrement hémisphérique à paroi musculeuse épaisse, présente deux groupes d'épines (fig. 4) :

- une série de quatorze à vingt petites épines de 25 à $28 \mu$ de longueur forme autour de l'ouverture centrale une couronne interrompue postérieurement. Ces épines ont un manche droit de 18 à $20 \mu$, une petite garde et une lame légèrement courbée 
terminée en pointe de 7 à $8 \mu$. Le manche est enfoncé jusqu'à la garde comprise dans le tissu musculaire de la paroi atriale, la lame est libre dans l'atrium ;

- une rangée de quatre à six épines centrales plus longues $(50-60 \mu)$ et plus robustes que les précédentes. Elles ont aussi un manche droit de $35-40 \mu$ avec une garde nette et une lame recourbée à pointe aiguë de 15-20 $\mu$. Elles sont serrées les unes contre les autres, les lames formant une sorte de peigne dans l'atrium, les manohes enfoncés dans la paroi atriale. En arrière de cette rangée d'épines et faisant face à leurs pointes on distingue une plaque irrégulière, légèrement sclérifiée. Cette plaque, formée par la paroi atriale, présente des rainures (une en face de chaque épine centrale). La répartition du nombre d'épines chez 66 individus examinés est résumée dans le tableau 1.

Tableau I

\begin{tabular}{|c|c|c|c|c|c|c|c|c|c|c|}
\hline \multirow{3}{*}{$\begin{array}{l}\text { Nombre d'épines ....... } \\
\text { Nombre d'individus ... }\end{array}$} & \multicolumn{7}{|c|}{ EPINES DE LA COURONNE } & \multicolumn{3}{|c|}{$\begin{array}{c}\text { EPINES } \\
\text { CENTRALES }\end{array}$} \\
\hline & 14 & 15 & 16 & 17 & 18 & 19 & 20 & 4 & 5 & 6 \\
\hline & 6 & 6 & 15 & 14 & 15 & 5 & 5 & 11 & 30 & 25 \\
\hline
\end{tabular}

APPAREIL GÉNITAL FEMELLE:

L'ovaire est situé dans la partie moyenne du corps. Il se présente comme une glande allongée, repliée sur elle-même. Il forme ainsi deux anses superposées, dirigées vers l'avant la première étant la plus dorsale (fig. 5). L'oviducte contourné qui lui fait suite est, chez certains individus, empli de spermatozoïdes et joue ainsi un rôle de réceptacle séminal. Le canal génito-intestinal qui se détache de l'oviducte aboutit dans la branche droite de l'intestin. Après avoir reçu le vitelloducte médian, l'oviducte forme sur le côté gauche du corps une anse postérieure marquée par une rosette de glandes de Mehlis. Au-delà, la branche ascendante, légèrement élargie, forme un ootype à parois glandulaires. L'utérus ventral, rectiligne, à lumière ciliée, aboutit à la partie postérieure de l'atrium génital au point où la couronne d'épines est interrompue. Les glandes vitellogènes forment deux larges bandes latérales entre les caecums du système digestif. Elles débutent, à l'avant au niveau de l'atrium génital et se réunissent, comme les branches digestives, dans la partie postérieure du corps. Les vitellogènes pénètrent dans la région médiane du hapteur, mais moins profondément que le diverticule intestinal qu'elles accompagnent. Les vitelloductes longitudinaux sont parfois visibles de chaque côté au niveau des branches digestives. Les vitelloductes transverses se détachent un peu en avant de l'ovaire. Dirigés vers l'arrière, ils se rejoignent sur la ligne médiane formant ainsi un $\mathrm{Y}$ parfois très net. Le vitelloducte impair va se jeter dans l'oviducte. L'ouverture vaginale dorsale, étirée transversalement, a ses bords plissés. Le canal vaginal plonge ventralement puis, sur la ligne médiane, se dirige vers l'arrière. Cette partie, parfois élargie, joue le rôle de réceptacle séminal. Le canal se divise alors en deux, 


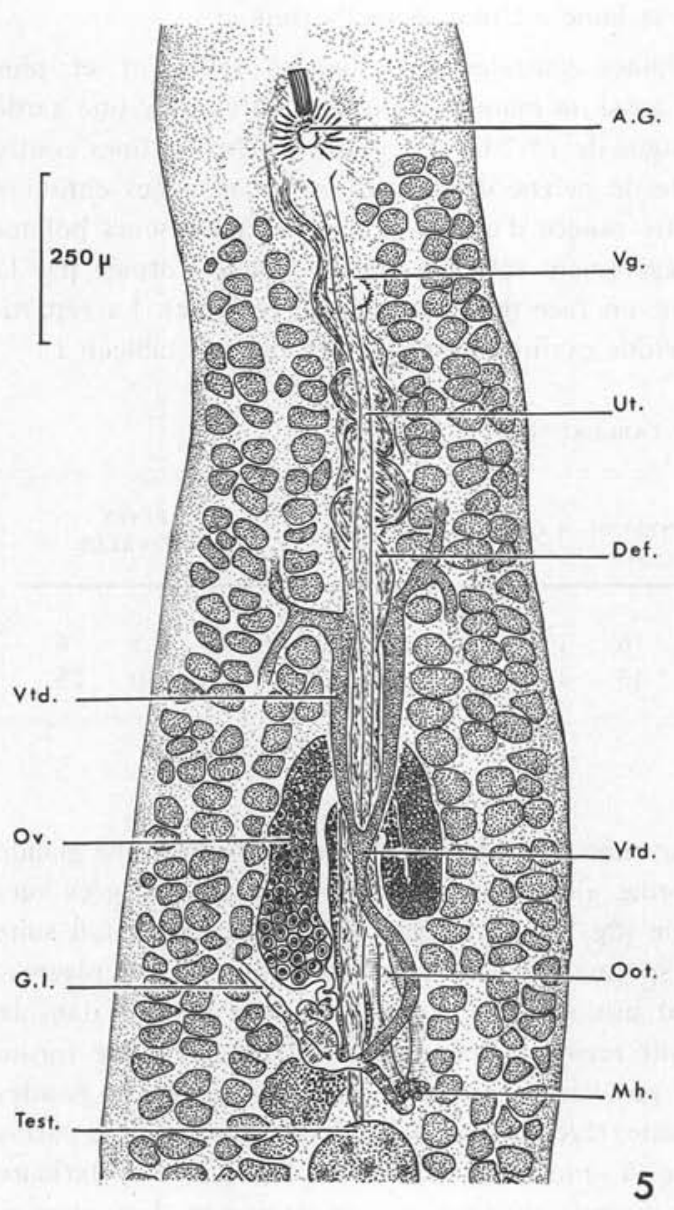

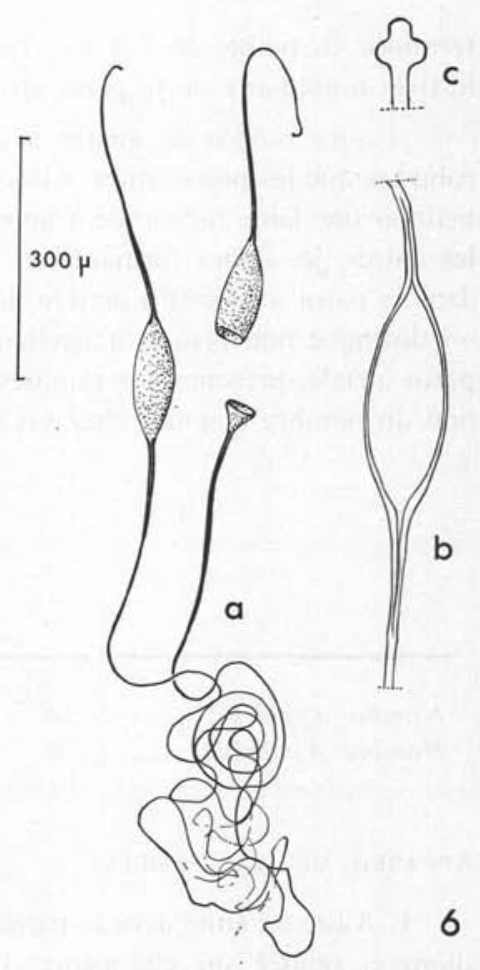

Fic. 5. - Microcotyle salpae Parona et Perugia, 1890. Système génital, vue ventrale. A.G. : atrium génital. - Def. : canal déférent. - G.I. : canal génitointestinal. - Mh. : glande de Mehlis, Oot. : ootype. - Ov. : ovaire. - Test. : testicule. - Ut. utérus. - Vg. : vagin. - Vtd. : vitelloductes.

FIG. 6. - Microcotyle salpae Parona et Perugia, 1890. a) Euf et œuf éclos. b) Détail de la coque. c) Extrémité très grossie du filament postérieur.

chaque conduit venant se jeter à l'origine d'un vitelloducte transverse. Les œufs utérins, dont la coque est assez transparente, nous ont montré dans quelques cas un ovule fécondé. C'est une sphère de $24 \mu$ avec un gros noyau de $8 \mu$ de diamètre et un nucléole parfois encore visible de $4 \mu$ de diamètre. La coque de l'œuf est jaune pâle au moment de la ponte mais el'e brunit progressivement dans l'heure qui suit. L'œuf pondu, fusiforme, mesure $150 \mu$ de longueur et $50 \mu$ de largeur avec un filament à chaque extrémité. L'un des filaments, que nous nommerons antérieur, car il sort le premier de l'atrium génital, est très long, fin en général, peletonné parfois assez régulièrement. Le filament opposé postérieur, assez épais, court ( $300 \mu$ environ) est terminé par un petit élargissement en forme de capuchon renversé (fig. 6). 


\section{Discussion.}

Malgré la brièveté de la description originale les détails concordants permettent d'assimiler l'espèce recueillie à Microcotyle salpae Parona et Perugia, 1890. L'hôte Box salpa est identique, le nombre de pinces du hapteur est le même. Dans les deux cas le vagin est une ouverture dorsale allongée transversalement avec les bords plissés. L'armature de l'atrium génital est identique. A ce propos Parona et Perugia signalent un cercle de seize crochets de $28 \mu$ et un faisceau de minuscules épines en bâtonnet. Le nombre et la taille de ces épines centrales ne sont pas donnés mais nous pouvons les déduire du dessin qui illustre la description. Nous comptons ainsi six épines centrales qui mesurent au moins le double des crochets de la couronne. Cela donne $56 \mu$; chiffre très voisin de celui $(60 \mu)$ que nous avons mesuré chez les organismes que nous avons étudiée. En outre, les prolongements postérieurs que Parona et Perugia ont dessiné audessous de ces épines centrales peuvent représenter la plaque sclérifiée que nous avons signalée. Il nous faut encore souligner que l'anatomie du système génital ressemble beaucoup à celle que les auteurs italiens ont donné pour Microcotyle sargi. Mais si la disposition des épines de l'atrium génital est voisine, le nombre et la taille de ces crochets sont très différents. Chez Microcotyle sargi, en particulier, les épines centrales sont nettement plus petites que les crochets de la couronne.

\section{Biologie.}

\section{Taux de parasitisme.}

Sur quarante saupes examinées immédiatement après leur capture en mer, nous avons trouvé dix individus non parasités (les animaux examinés après un séjour plus ou moins long en aquarium n'ont pas été comptés ici). Ceci correspond à un pourcentage global de parasitisme égal à $75 \%$. Bien que nous ayons trouvé des parasites pendant toute la durée de notre travail (une année), nous ne pouvons conclure que ce parasitisme n'est pas saisonnier. Une étude de plus longue durée nous permettra de le vérifier.

Nous avons aussi noté la taille de chaque poisson examiné. Les résultats nous ont permis d'affirmer que dans le golfe de Tunis les animaux les plus grands sont les plus parasités. Ainsi, nous n'avons pas trouvé de Microcotyle sur les saupes qui mesurent moins de $15 \mathrm{~cm}$ de longueur totale, par contre, celles qui ont entre 15 et $25 \mathrm{~cm}$ sont parasitées à presque $50 \%$, celles dont la taille dépasse $25 \mathrm{~cm}$ le sont à plus de $75 \%$.

Le nombre des parasites par poisson est très variable. Nous avons trouvé de un jusqu'à dix-sept parasites par hôte avec une moyenne de quatre à cinq. Sur un seul arc branchial nous avons compté jusqu'à dix parasites; la moyenne étant de deux à trois.

\section{Place du parasite.}

Nous avons noté la place exacte du parasite dans la cavité branchiale. Microcotyle salpae y est fixé par les pinces du hapteur, chaque pince enserrant une lamelle d'un même filament branchial. 
Ainsi, le parasite peut se rencontrer :

- soit sur le côté gauche, soit sur le côté droit du poisson,

- soit sur le premier, le deuxième, le troisième ou le quatrième arc branchial,

- soit sur le filament antérieur, soit sur le filament postérieur,

- soit sur la face supérieure, soit sur la face inférieure de ce filament,

- soit dans la région dorsale, soit dans la région ventrale de l'arc.

A ce sujet, nous avons suivi Llewellyn et subdivisé l'arc branchial en cinq parties à peu près égales numérotése de $\mathrm{I}$ à $\mathrm{V}$ à partir de la région dorsale (fig. 7). Nous pouvons ainsi représenter chaque arc branchial par un rectangle divisé en dix cases, 5 représentant les filaments (hémibranchie) antérieurs et cinq pour les filaments (hémibranchie)

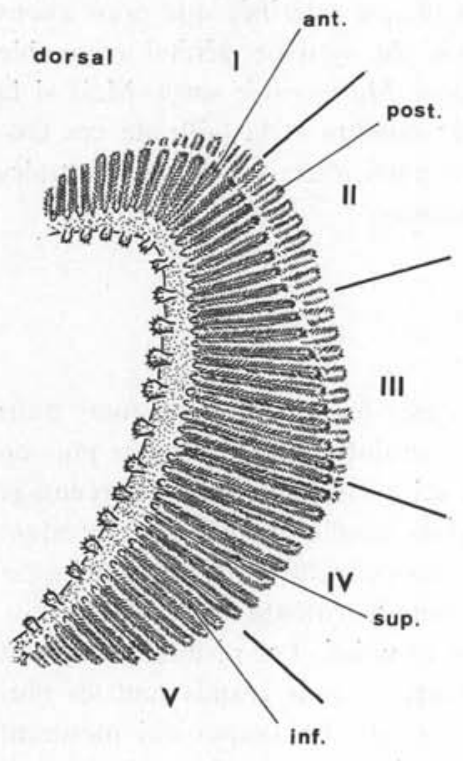

ventral
FIG. 7. - Représentation schématique d'un arc branchial gauche. I, II, III, IV et V : régions définies arbitrairement sur 1'arc; ant.: filaments (Hémibranchie) antérieurs; post. : filaments (Hémibranchie) postérieurs; sup.: face supérieure d'un filament; inf.: face inférieure d'un filament.

postérieurs. Les cases du schéma numérotées de I à V du haut vers le bas correspondent aux parties définies sur l'arc. Dans chaque case nous inscrivons le nombre de parasites trouvés dans cette position. Les chiffres dans les deux cases inférieures $\mathbf{A}$ et $\mathbf{P}$ représentent le nombre total de parasites trouvés sur les filaments antérieurs et sur les filaments postérieurs. Le nombre médian $\mathrm{T}$ est le nombre total de parasites récoltés sur l'arc considéré. Dans ce travail la position face inférieure-face supérieure du filament n'a pas été notée. Cette position n'ayant de véritable intérêt que dans l'étude des formes nettement dissymétriques. 
Schéma représentant un arc branchial

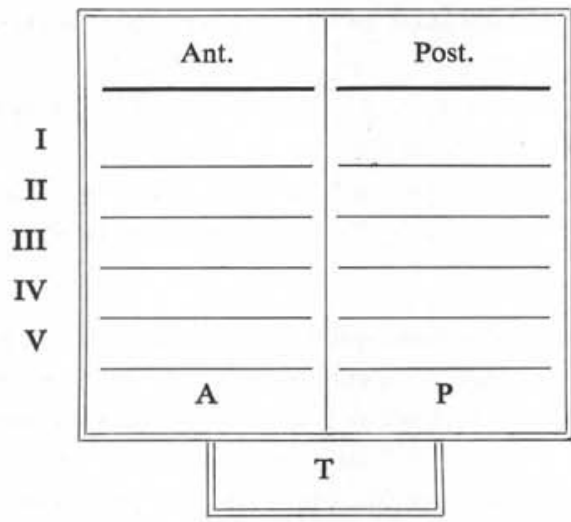

Pour trente poissons parasités nous avons noté la répartition suivante dans le tableau II
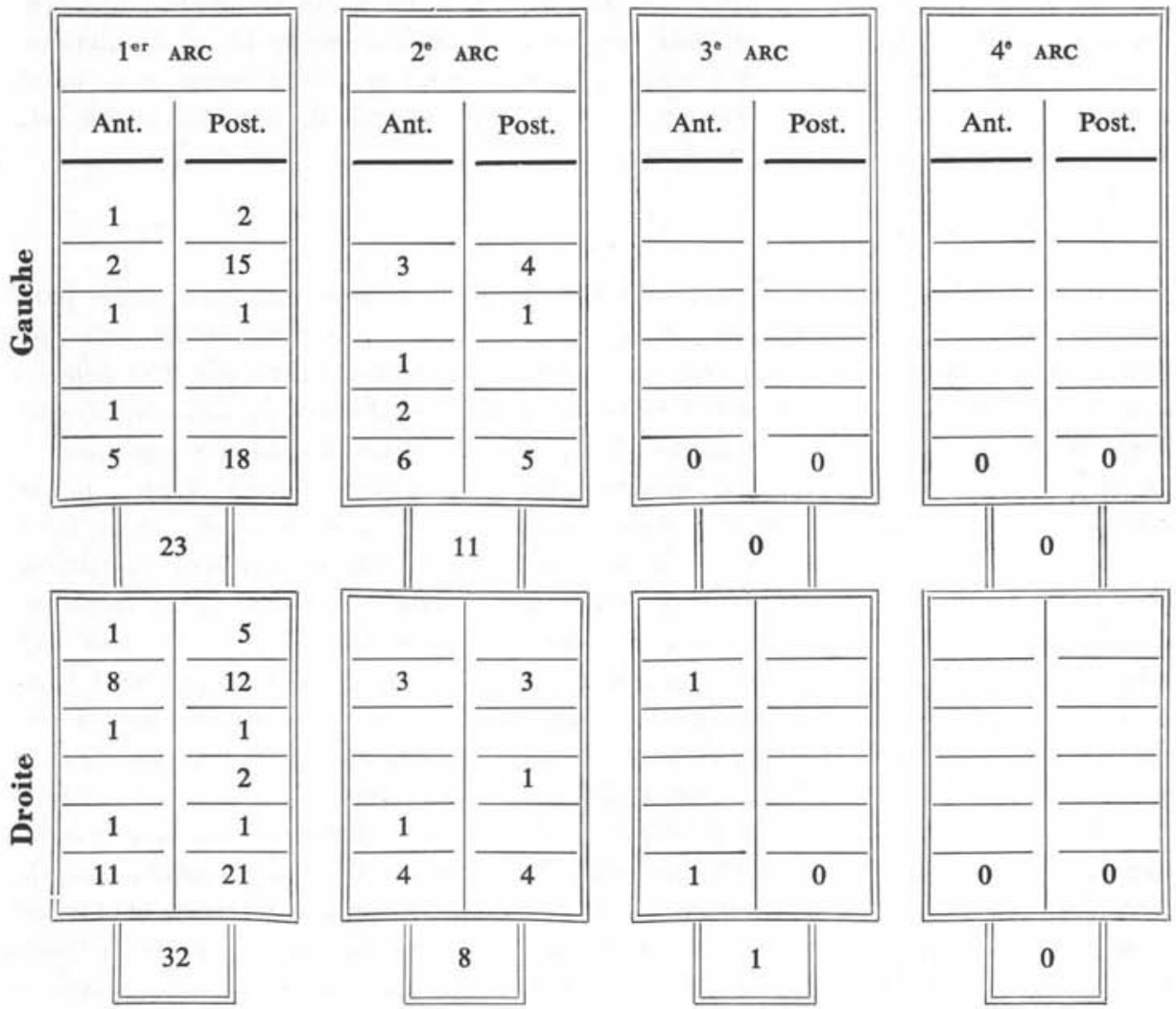

TABleaU II 
L'étude du tableau II nous permet de dire que les parasites :

- se trouvent aussi bien dans la cavité branchiale droite (34) que dans la cavité branchiale gauche (41),

- se placent surtout sur l'arc 1 (55) et l'arc 2 (19), très rarement sur l'arc 3 (1) mais jamais sur le quatrième,

- se rencontrent en majorité (48) soit $64 \%$ sur les filaments postérieurs,

- se trouvent le plus souvent $(68 \%)$ en position II c'est-à-dire sur la partie dorsale de l'arc.

En outre, nous avons remarqué que le plus souvent il n'y a de parasites sur l'arc 2 que s'il y en a déjà sur l'arc 1 . Ceci est très net dans le seul cas où nous avons trouvé un parasite sur l'arc 3 et où il y avait déjà deux parasites sur le deuxième arc et deux parasites sur le premier arc du même côté. Ceci nous permet de dire qu'il y a sans doute une concurrence entre les parasites pour la place préférentielle qui est le premier arc. Si cet arc est déjà parasité les autres parasites se placeront sur le deuxième arc et éventuellement si besoin est, sur le troisième arc.

En général, le parasite est fixé aux lamelles du filament postérieur, le corps proprement dit étant placé entre les deux rangées de filaments vers le filament antérieur. C'est là la position définie par Llewellyn comme étant le plus propice pour lutter contre le courant d'eau. Cependant, ici, il n'y a pas dissymétrie du parasite comme cela se rencontre chez Gastrocotyle trachuri.

\section{Développement.}

Les Microcotyle salpae adultes détachés du filament branchial sont placés dans une coupelle emplie d'eau de mer. Après quelques heures, ils pondent des œuts quı généralement sont groupés en paquets grâce à leurs filaments enchevêtrés. Ces paquets d'œufs sont alors prélevés et placés dans des petits cristallisoirs de $125 \mathrm{~cm}^{3}$ emplis d'eau de mer filtrée et additionnée de $15 \mathrm{mg}$ /litre de Streptomycine qui agit comme antibiotique sur les bactéries qui, souvent, gênent ce genre d'élevage. Cette eau est renouvelée quotidiennement. Placés dans ces conditions, les œufs n'ont jamais éclos naturellement, pourtant une dizaine de jours après la ponte, ils montrent au microscope une larve vivante que l'on voit par transparence, bouger à l'intérieur de la coque.

Nous nous sommes alors aperçu qu'une action mécanique pouvait avoir une influence sur l'éclosion. En effet, lorsque nous agitons après le huitième jour l'eau d'élevage, avec le jet d'un compte-gouttes, afin d'en réaliser l'oxygénation, nous avons obtenu quelques larves. Mais l'examen des œufs restants nous a prouvé qu'une grande majorité étaient embryonnés et contenaient une larve vivante.

Nous avons alors pensé qu'il s'agissait d'un facteur chimique identique à celui que Euzet et Raibaut (1960) ont signalé dans l'éclosion de l'œuf de Squalonchocotyle torpedinis (Hexabothriidae). Nous avons réalisé l'élevage comme précédemment, mais le septième jour lorsque nous avons commencé à observer des larves vivantes à l'intérieur de l'œuf, nous avons ajouté à l'eau d'élevage du mucus obtenu en raclant avec le 
dos d'un scalpel les branchies d'une Saupe. Nous avons alors obtenu près de $50 \%$ d'éclosions et $75 \%$ environ après agitation de l'eau.

L'adulte se rencontre en général sur les branchies de Box salpa, mais nous l'avons une fois récolté sur le Sars (Diplodus sargus). Pour savoir si le facteur chimique mis en cause était spécifique, nous avons alors tenté une expérience plus précise.

Nous avons réparti la ponte d'un même jour en trois lots, chaque lot contenant 40 œufs.

Lot $\mathrm{n}^{\circ} 1$ : Lot témoin. Il a été placé dans un bac contenant de l'eau de mer filtrée additionnée d'antibiotiques.

Lot $\mathrm{n}^{\circ} 2$ : Les œufs de ce lot ont été mis en élevage comme précédemment, mais dans le bac nous avons ajouté à partir du sixième jour, une dizaine de gouttes de l'eau d'un petit aquarium où vivaient des Saupes depuis plus de 48 heures. La dose a été augmentée et portée à 20 gouttes à partir du dixième jour.

Lot $\mathrm{n}^{\circ} 3$ : Les œufs de ce lot ont été placés comme le lot témoin dans un bac, mais on a ajouté à partir du sixième jour, de l'eau d'un petit aquarium où vivaient des Sars depuis plus de 48 heures. La même dose a ici aussi été augmentée après le dixième jour de la même façon.

A partir du huitième jour, nous avons observé des sorties de larves et nous avons noté quotidiennement le nombre d'éclosions dans chaque lot.

Nous résumons dans le tableau III ces résultats :

\begin{tabular}{|c|c|c|c|}
\hline & Lot 1 & Lot 2 & Lot 3 \\
\hline JOURS APRÈS LA PONTE : & & & \\
\hline $8 \quad \ldots \ldots \ldots \ldots \ldots \ldots$ & 0 & 8 & 1 \\
\hline $9 \quad \ldots \ldots \ldots \ldots \ldots \ldots$ & 0 & 4 & 1 \\
\hline $10 \quad \ldots \ldots \ldots \ldots \ldots \ldots$ & 0 & 0 & 0 \\
\hline $\begin{array}{l}\text { AUGMENTATION DE LA DOSE } \\
\text { AJOUTÉE: }\end{array}$ & & & \\
\hline $11 \quad \ldots \ldots \ldots \ldots \ldots \ldots$ & 0 & 12 & 0 \\
\hline $12 \ldots \ldots \ldots \ldots \ldots \ldots$ & 0 & 0 & 0 \\
\hline Agitation $\quad \ldots \ldots \ldots \ldots \ldots$. & 1 & 5 & 2 \\
\hline
\end{tabular}

TABLEAU III

A partir du douzième jour et malgré une nouvelle augmentation de la quantité d'eau ayant contenu des poissons, nous n'avons pas obtenu de nouvelles éclosions.

Par contre, en agitant l'eau, nous avons pu obtenir quelques larves comme lors de nos premières expériences, ces larves ont cependant été plus nombreuses dans les bacs 2 et 3 . 
D'après ces expériences, nous pouvons penser qu'il existe, pour déclencher l'éclosion de l'œuf de Microcotyle salpae, un facteur chimique, que l'action de ce facteur est très importante et qu'elle paraît spécifique. Mais il doit s'y ajouter une action mécanique dont il ne faut pas négliger le rôle.

\section{Description de la larve.}

L'éclosion de l'œuf se fait par ouverture d'un opercule qui se détache à $50 \mu$ environ du côté du long filament. De l'œuf sort une larve non ciliée qui ne nage pas et se fixe par sa partie antérieure sur le fond du bac d'élevage.

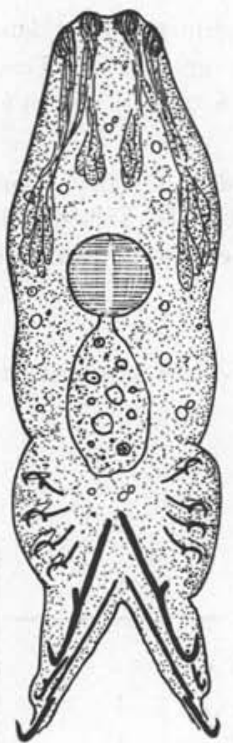

a

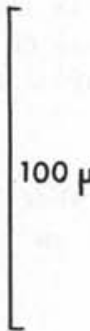

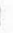

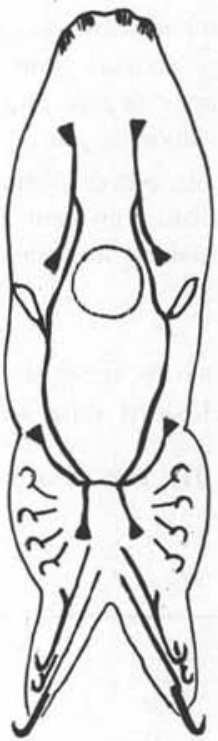

b
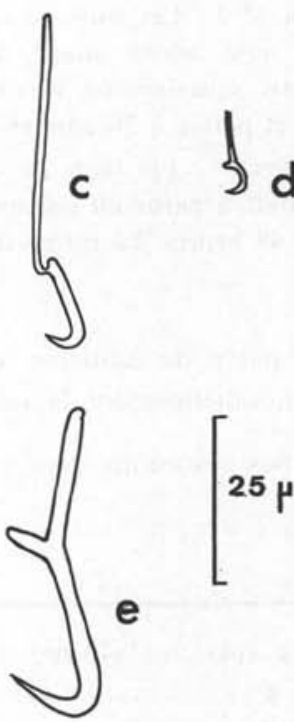

Fig. 8. - Microcotyle salpae Parona et Perugia, 1890. a) larve non ciliée d'après l'observation sur le vivant ; $b$ ) schéma de la disposition du système excréteur ; c) crochet I (en fléau); $d$ ) crochet II (postéro-latéral) ; e) Hamuli

La larve aplatie dorso-ventralement, mesure environ $200 \mu$ de longueur et $70 \mu$ de largeur. Il n'y a pas de taches oculaires (fig. $8 a$ ).

A l'arrière du corps, le hapteur forme deux lobes postérieurs égaux légèrement divergents qui, dès la sortie de l'œuf ont des mouvements d'allongement et d'essai de fixation. La forme et la disposition des crochets que l'on trouve dans ce hapteur sont caractéristiques et semblables à celles que l'on a signalé chez les autres représentants des Microcotylidae (Euzet 1958). Nous avons ainsi de chaque côté du plan de symétrie, et d'arrière et avant selon la terminologie définie par Llewellyn dans son travail de 1960. 
Crochet I (postérieur). C'est un crochet en fléau avec un manche rectiligne de $40 \mu$ de longueur. La lame de $13 \mu$ incurvée dans sa partie distale se termine en pointe aiguë (fig. $8 c$ ).

Crochet II (postéro-latéral). Crochet de $13 \mu$ de longueur avec un manche de $8 \mu$ et une lame de $5 \mu$. C'est un crochet «à conducteur » qui joue dans une « ogive » ou «domus * (fig. $8 d$ ).

Hamuli : Ce crochet puissant mesure 48 à $50 \mu$ de longueur totale. Le manche de $22 \mu$ est aussi long que la garde. La lame recourbée mesure $26 \mu$ (fig. $8 e$ ).

Crochets III, IV, V, VI (latéraux). Ils mesurent $13 \mu$ de longueur et leur morphologie est identique à celle du crochet II.

Chaque lobe postérieur que nous avons signalé plus haut contient un crochet I. un crochet II et un hamuli. Les crochets III, IV, V et VI sont situés en arc de cercle de chaque côté du hapteur.

A la partie antérieure du corps légèrement lobée débouchent quatre amas glandulaires. A $60 \mu$ environ de cette extrémité, le pharynx médian se présente sous la forme d'une sphère musculeuse de $25 \mu$ environ de diamètre. Il se continue par un intestin sacciforme contenant de petites gouttelettes d'aspect huileux. Le système excréteur a une disposition identique à celle décrite par Euzet et Cauwet chez la larve de Polyarbris diplodi (Microcotylidae). Il comprend de chaque côté deux protonéphridies antérieures et deux protonophridies postérieures. Les flammes antérieures comme les flammes postérieures donnent des canalicules qui se rejoignent pour former un canal antérieur et un canal postérieur. De leur union résulte donc un tronc excréteur cilié qui débouche dorsalement à l'extérieur au niveau du pharynx. La partie terminale de ce tronc se renfle parfois en une vésicule excrétrice (fig. $8 b$ ).

\section{Conclusion.}

Actuellement, on connaît seulement deux monogènes dont la larve non nageante n'a pas le corps recouvert de cellules ciliées. Ce sont:

- Acanthocotyle lobianchi parasite de divers raies (Raja clavata, R. montagui, $R$. brachyura, $R$. naevus) dont la larve brièvement décrite par Llewellyn en 1957 a été revue et bien étudiée par Kearn en 1967.

- Squalonchocotyle torpedinis parasite de Torpedo marmorata dont la larve décrite par Euzet en 1957 a été retrouvée et étudiée par Euzet et Raibaut en 1960.

Comme l'a fait remarquer Llewellyn Raies et Torpilles passent la plus grande partie de leur vie sur le fond de la mer et il est alors possible que l'absence de cils chez la larve soit liée à cet habitat sédentaire de l'hôte. Les œufs embryonnés attendent sur le fond qu'un hôte passe à proximité (cas d'Acanthocotyle parasite externe) ou d'être avalé et passer dans la cavité branchiale avec le courant d'eau respiratoire (Squalonchocotyle torpedinis parasite branchial).

Il faut cependant souligner qu'un autre monogène Entobdella soleae qui parasite un pleuronectidae (Solea solea) ayant une vie tout aussi sédentaire, a gardé une larve nageante ciliée. 
Les Saupes sont des poissons vivant en pleine eau et l'hypothèse précédente ne peut s'app'iquer à cette biologie. Mais; les Saupes sont des animaux herbivores qui mangent des algues. Ce comportement est particulièrement net en captivité où on les voit brouter les parois de l'aquarium où elles vivent.

Il se peut que les œufs retenus par leurs filaments aux algues passent dans la cavité buccale au moment du repas et aussi avec le courant d'eau respiratoire. L'éclosion est alors facilitée nar les deux facteurs que nous avons mis en évidence et la larve passant dans la cavité branchiale se fixe immédiatement sur la branchie où elle va se développer.

Nous espérons apporter ultérieurement les preuves expérimentales qui confirmeront cette hypothèse.

\section{Bibliographie}

EUZET (L.), 1957. - Recherches sur les Monogenoidea parasites de poissons marins. Ann. Parasit. Hum. Comp., 32 (5-6) : 469-481, fig. 1-17.

-, 1957. - Larves gyrodactyloïdes nageantes de quelques Microcotylidae (TrematodaMonogenea). Bull. Soc. Neuch. Sci. Nat., 80:187-194, fig. 1-24.

-, 1958. - Sur le développement post-larvaire des Microcotylidae (Monogenoidea, Polyopisthocotylea). Bull. Soc. Neuch. Sci. Nat., 81 : 79-84, fig. 1-7.

- et Cauwet (A.), 1967. - Polyabris diplodi, n. g., n. sp. (Monogenea, Microcotylidae) parasite de Téléostéens du genre Diplodus (Sparidae). Bull. Mus. Nat. Hist. Nat., 39 (1) : 213-220, fig. 1-6.

- et MARC (A.), 1963. - Microcotyle donavini Van Beneden et Hesse 1863, espèce type du genre Microcotyle Van Beneden et Hesse 1863. Ann. Parasit. Hum. Comp., 38 (6) : 875-886, fig. 1-14.

- et Raibaut (A.), 1960. - Le développement post-larvaire de Squalonchocotyle torpedinis (Price 1942). Bull. Soc. Neuch. Sci. Nat., 83: 101-108, fig. 1-6.

KeARN (G. C.), 1967. - The life-cycles and larval development of Some Acanthocotylides (Monogenea) from Plymouth rays. Parasitology, 57: 157-167, fig. 1-6.

Llewellyn (J.), 1957. - The larvae of some monogenetic trematodes of Plymouth fishes. J. Mar. Biol. Ass. U.K., 36: 243-259, fig. 1-28.

-, 1959. - The larval development of two species of Gastrocotylid trematodes parasites from the gills of Trachurus trachurus. J. Mar. Biol. Ass. U.K., 38 : 461-467, fig. 1-9.

-, 1960. - Amphibdellid (Monogenea) parasites of electric rays (Torpedinae). J. Mar. Biol. Ass. U.K., 39: 561-589, fig. 1-28, pl. 1-3.

-, 1963. - Larvae and larval development of Monogeneans. Advances. Parasit., $1^{\circ}: 287$ 326, fig. 1-15.

Mac Callum (G. A.) et Mac Callum (W. G.), 1913. - Four species of Microcotyle; $M$. pyrographorus, M. macroura, M. eueides and M. acanthophallus. Zool. Jb. (Syst.), 34 : 223-244. 
Meserve (F. G.), 1938. - Some monogenetic trematodes from the Galapagos Islands and the neighbouring pacific. Allan. Hancock. Paci. Expe. (Univ. S. Calif. Publ.), 2: 27-89, fig. 1-85.

Palombi (A.), 1949. - Trematodi d'Italia. Parte I. Trematodi monogenetici. Archiv. Zool. Italiano, 34 : 203-408, fig. 1-89.

Parona (C.) et Perugia (A.), 1890. - Contribuzione per una monografia del genere Microcotyle (Res Ligusticae 14). Ann. mus. Stor. nat. Genova. ser. 2, $10: 173-220$, fig. 1-34.

Sproston (N. G.), 1946. - A synopsis of the monogenetic trematodes. Trans. Zool. Soc. London, 25 : 185-600, fig. 1-118.

Yamaguti (S.), 1963. - Systema helminthum IV. Monogenea and Aspidocotylea. Interscience publ. (N.Y.), 1-699, fig. 1-898. 